\title{
Video Coding of Various Decomposition with Encoding Techniques
}

\author{
Nithin.S.S, L.Padma Suresh
}

\begin{abstract}
In a period of past ten years, Compression or coding of video has been elaborated as a vital role in the part of total communication. Multi resolutions techniques play a vital part in the coding of image and video. Wavelet and curve let transform are one of the popular and efficient technique in video coding. In my paper, I am operate to implement various coding based methods with (EWT)empirical wavelet transform, different wavelets, curvelets with various encoding techniques. They are video coding with EWT and H.264(VEWH), video coding with EWT and LZW(VEWL), video coding with EWT and Huffman with SPIHT(VEWHS), video coding with mexican hat wavelet transform and SPIHT(VMWS), video coding with dual tree wavelet and SPIHT(VDTS), video coding with $3 D$ dual tree wavelet transform and SPIHT(V3DTS), video coding with curvelet transform and SPIHT(VCTS) and video coding with dual tree complex wavelet fractional transform and modified SPIHT(VDTCWFS). Next we implement these methods with the assist of matlab 2014 and 2015, and then analyze these techniques by using PSNR and compression ratio.
\end{abstract}

Index Terms: (EWT) Empirical wavelet transforms, H.264, VEWH, LZW, VEWL, SPIHT, VEWHS, VMWS, VDTS, V3DTS, VCTS, VDTCWFS, PSNR

\section{INTRODUCTION}

The communication/transmit of a huge amount data of video is processed only by restricting the size but it is attained by the use of video compression techniques. Different areas such as genetics, astronomy, video communications, remote sensing and medical field is the main application of (DIP) digital image processing. deliberate to provide a digital description of a signal. When the input datas like mage, video, and audio signals are in compressed form, then we can operate this in various demands on the idustry or customers. Most of the operations related to image processing need compressed or coded data for storage and other operations. Predominantly, majority signals are maintained in the time domain condition and the processing of signal is emerged on in order to get more information. The signals become tranmuted by various mathematical analysis functions for this basis. The wavelet transform is a wave structure which has productively restricted duration that has a minimum utility of zero. The phrase wavelet approaches from the fact that wave up and down over the axis.

The wavelets have glowing outcomes in the domain of video processing, and have been used to find many

Revised Manuscript Received on July 22, 2019.

Mr.Nithin S.S, Research Scholar, Department of Electronics and Communication, Noorul Islam University, Tamil Nadu, ,India

Dr. L.Padma Suresh, Professor and Principal,Department of Computer Science, Baselios Mathews II College Of Engineering, Sasthamkotta.Kerala,India

problems/applications such as video/image compression or coding and restoration. The figure 1 represents block representation of basic compression/coding of the video or image. Multi resolutions techniques play a vital part in the coding of image and video. Wavelet and curve let transform are one of the popular and efficient technique in video coding. In my paper, I am operate to implement various coding based methods with (EWT)empirical wavelet transform, different wavelets ,curvelets with various encoding techniques. They are 1) video coding with EWT and H.264(VEWH), 2)video coding with EWT and LZW(VEWL), 3)video coding with EWT and Huffman with SPIHT(VEWHS), 4)video coding with mexican hat wavelet transform and SPIHT(VMWS), 5)video coding with dual tree wavelet and SPIHT(VDTS), 6)video coding with $3 \mathrm{D}$ dual tree wavelet transform and SPIHT(V3DTS), 7)video coding with curvelet transform and SPIHT(VCTS) and 8)video coding with dual tree complex wavelet fractional transform and modified SPIHT(VDTCWFS). Next we implement these methods with the assist of matlab 2014 and 2015, and then analyze these techniques by using PSNR and compression ratio.

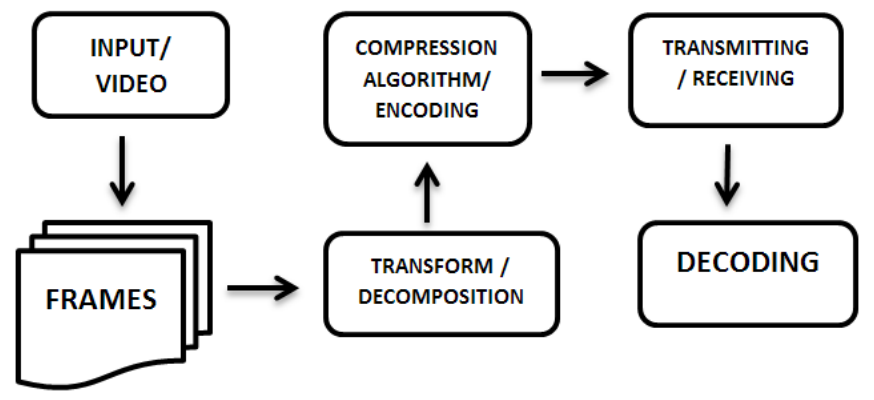

Fig 1: Block representation of basic compression/coding

\section{VIDEO CODING WITH EWT AND H.264(VEWH)}

In this method, compression or coding was executed with the help of empirical wavelet transform and H.264. EWT is mainly used for decomposite the data consider to the information in that. So it is conventional to many approaches. In this work/ implementation, we decomposite the data by the usage of emperical wavelet transform and next encode it by H.264 coding. The figure 2 denotes the block presentation of this implementation. Here first the video is converted in to frames by using matlab 2015.Then the decomposition is done or processed by the use of emperical wavelet transform. The compression/coding process is done with the help of H.264 coding. Then we can easily transmit this data. The reconstruction part is done with the help of inverse transform and decoder. The performance

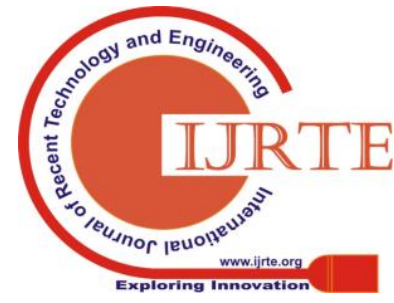


analysis of theis system is done with the help of PSNR, SSM and compression ratio.

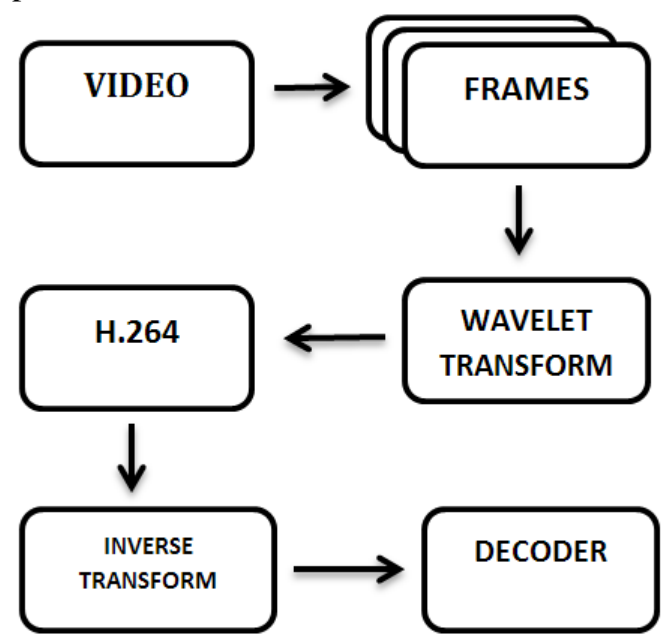

Fig 2: Block representation of video coding with EWT and H.264(VEWH)

\section{VIDEO CODING WITH EWT AND LZW(VEWL)}

In this method, compression or coding was executed with the help of empirical wavelet transform and LZW. EWT is mainly used for decomposite the data consider to the information in that. So it is conventional to many approaches. In this work/ implementation, we decomposite the data by the usage of emperical wavelet transform and next encode it by LZW coding. The figure 3 denotes the block presentation of this implementation. Here first the video is converted in to frames by using matlab 2015.Then the decomposition is done or processed by the use of emperical wavelet transform. The compression/coding process is done with the help of LZW coding. Then we can easily transmit this data. The reconstruction part is done with the help of inverse transform and decoder. The performance analysis of theis system is done with the help of PSNR, SSM and compression ratio.

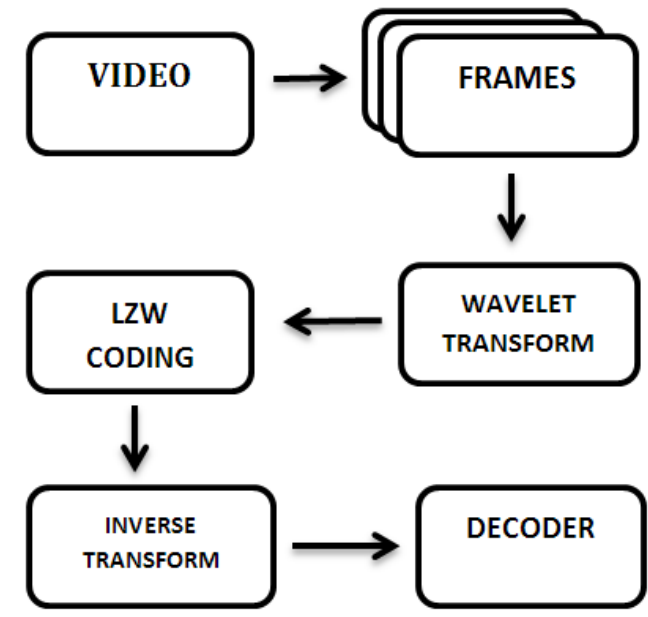

Fig 3: Block representation of video coding with EWT and LZW(VEWL)

\section{VIDEO CODING WITH EWT AND HUFFMAN WITH SPIHT(VEWHS)}

In this method, compression or coding was executed with the help of empirical wavelet transform and huffman with SPIHT. EWT is mainly used for decomposite the data consider to the information in that. So it is conventional to many approaches. In this work/ implementation, we decomposite the data by the usage of emperical wavelet transform and next encode it by huffman and SPIHT coding. The figure 4 denotes the block presentation of this implementation. Here first the video is converted in to frames by using matlab 2015.Then the decomposition is done or processed by the use of emperical wavelet transform. Then based on the frequencies it is divided in to two parts, low frequency and high frequency. Huffman coding is used for encoding low frequency frames and SPIHT is used for encoding high frequency frames. Then we can easily transmit this data. The reconstruction part is done with the help of inverse transform and decoder. The performance analysis of this system is done with the help of PSNR, SSM and compression ratio.

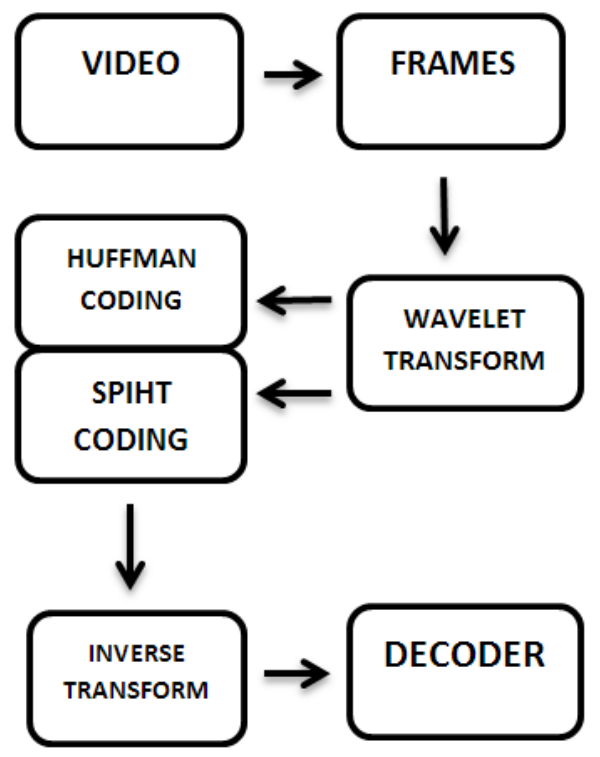

Fig 4: Block diagram of video coding with EWT and Huffman with SPIHT(VEWHS)

\section{VIDEO CODING WITH MEXICAN HAT WAVELET TRANSFORM AND SPIHT(VMWS)}

In this method, compression or coding was executed with the help of mexican hat wavelet transform and SPIHT. In this work/ implementation, we decomposite the data by the usage of mexican hat wavelet transform and next encode it by SPIHT coding. The figure 5 denotes the block presentation of this implementation. Here first the video is converted in to frames by using matlab 2015.Then the decomposition is done or processed by the use of mexican hat wavelet transform. The compression/coding process is done with the help of SPIHT coding. Then we can easily transmit this data. The reconstruction part is done with the help of inverse transform and decoder. The performance analysis of this system is done with the help of PSNR, SSM and compression ratio.

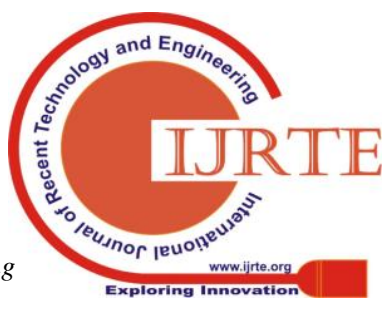




\section{VIDEO CODING WITH DUAL TREE WAVELET AND SPIHT(VDTS)}

Dual tree wavelet is a very important technique and it is mainly used in image processing. In this method, compression or coding was executed with the help of dual tree wavelet transform and SPIHT. DTW is mainly used for decomposite the data consider to the information in that. So it is conventional to many approaches. In this work/ implementation, we decomposite the data by the usage of dual tree wavelet transform and next encode it by SPIHT coding.

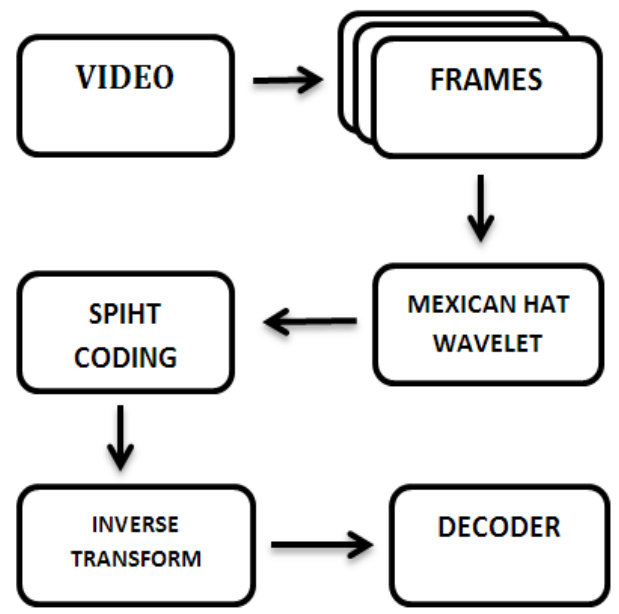

Fig 5: video coding with mexican hat wavelet transform and SPIHT(VMWS)

The figure 6 denotes the block presentation of this implementation. Here first the video is converted in to frames by using matlab 2015.Then the decomposition is done or processed by the use of dual tree wavelet transform. The compression/coding process is done with the help of SPIHT coding. Then we can easily transmit this data. The reconstruction part is done with the help of inverse transform and decoder. The performance analysis of theis system is done with the help of PSNR, SSM and compression ratio.

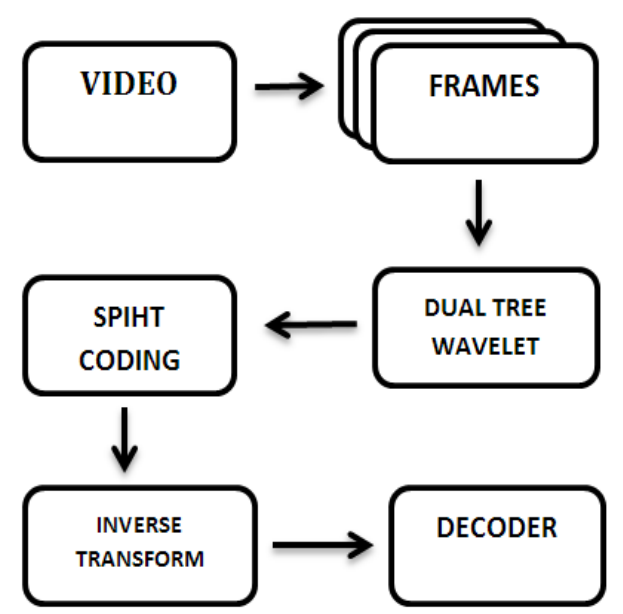

Fig 6: Block of video coding with dual tree wavelet and SPIHT(VDTS)

\section{VIDEO CODING WITH 3D DUAL TREE WAVELET TRANSFORM AND SPIHT(V3DTS)}

In this method, compression or coding was executed with the help of 3D dual tree wavelet transform and SPIHT. 3D-DTW is mainly used for decomposite the data consider to the information in that. In this work/ implementation, we decomposite the data by the usage of $3 \mathrm{D}$ dual tree wavelet transform and next encode it by SPIHT coding. The figure 7 denotes the block presentation of this implementation. Here first the video is converted in to frames by using matlab 2015.Then the decomposition is done or processed by the use of 3D dual tree wavelet transform. The compression/coding process is done with the help of SPIHT coding. Then we can easily transmit this data. The reconstruction part is done with the help of inverse transform and decoder. The performance analysis of this system is done with the help of PSNR, SSM and compression ratio.

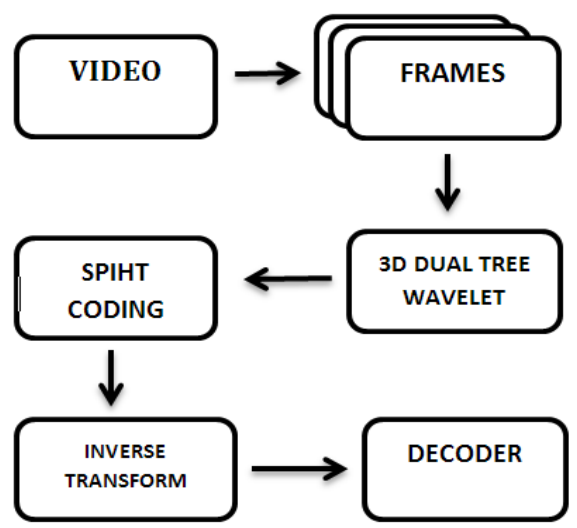

Fig 7: Block of video coding with 3D dual tree wavelet transform and SPIHT(V3DTS)

\section{VIDEO CODING WITH CURVELET TRANSFORM AND SPIHT(VCTS)}

Curvelets are purposed to hold curves operate only a small number of quantity. Therefore the Curvelet hold curve discontinuities. In this work/ implementation, we decomposite the data by the usage of 3D dual tree wavelet transform and next encode it by SPIHT coding. The figure 8 denotes the block presentation of this implementation. Here first the video is converted in to frames by using matlab 2015.Then the decomposition is done or processed by the use of curvelet transform. The compression/coding process is done with the help of SPIHT coding. Then we can easily transmit this data. The reconstruction part is done with the help of inverse transform and decoder. The performance analysis of theis system is done with the help of PSNR, SSM and compression ratio. 


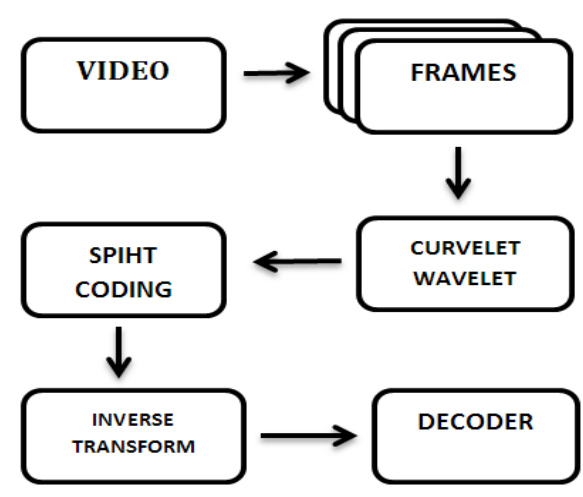

Fig 8: Block of video coding with curvelet transform and SPIHT(VCTS)

\section{VIDEO CODING WITH DUAL TREE COMPLEX WAVELET FRACTIONAL TRANSFORM AND MODIFIED SPIHT(VDTCWFS).}

Dual tree wavelet is a very important technique and it is mainly used in image processing. In this method, compression or coding was executed with the help of dual tree complex fractional transform wavelet transform and modified SPIHT. In this work/ implementation, we decomposite the data by the usage of dual tree complecx wavelet fractional transform and next encode it by modifiedSPIHT coding. The figure 9 denotes the block presentation of this implementation. Here first the video is converted in to frames by using matlab 2015.Then the decomposition is done or processed by the use of dual tree complecx wavelet fractional transform. The compression/coding process is done with the help of modified SPIHT coding. Then we can easily transmit this data. The reconstruction part is done with the help of inverse transform and decoder. The performance analysis of theis system is done with the help of PSNR, SSM and compression ratio. Results shows that the performance is better than previous methods.

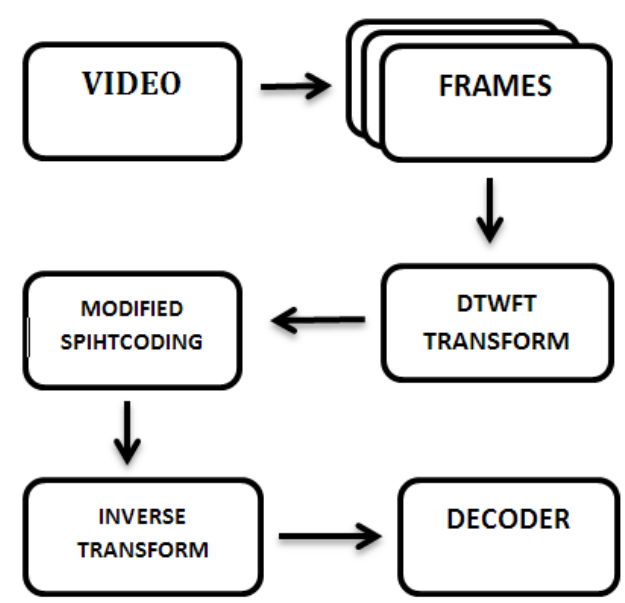

Fig 9: Block of video coding with dual tree complex wavelet fractional transform and modified SPIHT(VDTCWFS).

\section{X.PERFORMANCE ANALYSIS AND RESULTS}

In our approach, we analyze eight different ways of video coding. Figure10 represents the performance analysis of these methods based on PSNR and MSE. The eight different ways of my implementation includes 1) video coding with EWT and H.264(VEWH), 2)video coding with EWT and LZW(VEWL), 3)video coding with EWT and Huffman with SPIHT(VEWHS), 4)video coding with mexican hat wavelet transform and SPIHT(VMWS), 5)video coding with dual tree wavelet and SPIHT(VDTS), 6)video coding with 3D dual tree wavelet transform and SPIHT(V3DTS), 7)video coding with curvelet transform and SPIHT(VCTS) and 8)video coding with dual tree complex wavelet fractional transform and modified SPIHT(VDTCWFS).
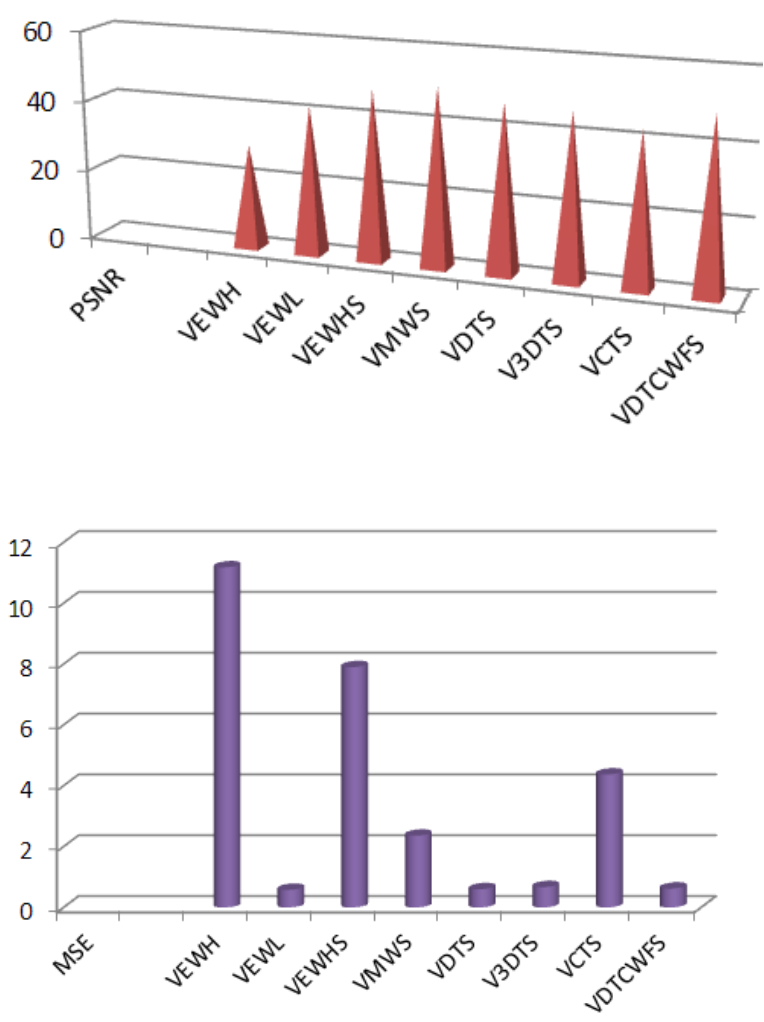

Fig 10: Performance analysis of various methods

In my implementation VDTCWFS got comparatively better outputs than other implementations. Other methods are also having good performance. From that I choose VDTCWFS as a better option for video coding. The figure 10 shows results of VDTS and VDTCWFS.

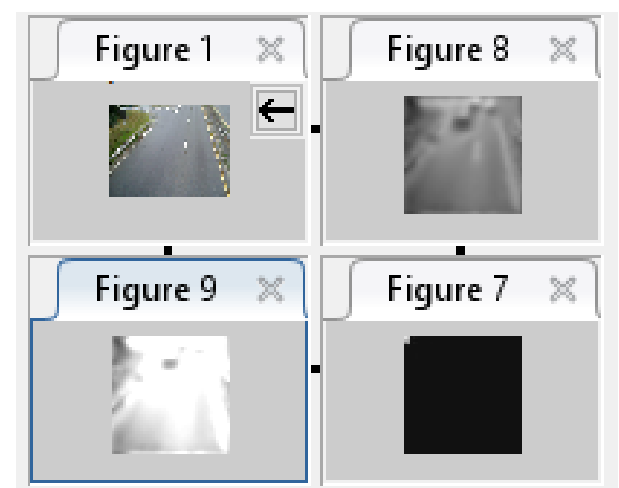




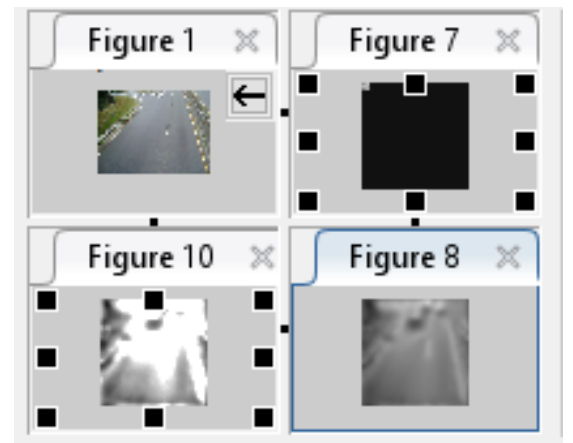

Fig 10: Results of VDTS and VDTCWFS

\section{CONCLUSION}

There are variety of methods are accessible in the present market for compressing the video. Here I also analyse different ways of compresion methods. We compress the video by using EWT,mexican hat, dual tree,3D dual tree,curvelet and dual tree fractional transform. By comparing the PSNR and MSE video coding using VDTCWFS got comparatively better outputs than other implementations.

\section{REFERENCES}

[1]. Mr.Nithin.s.s, Dr.L.Padma Suresh , N.SubashDaubechies, , 1998, "Wavelet based Video Compression Using Various Encoding Techniques: A Relative Study", International Journal of Engineering \& Technology, 7 (3.24) (2018) 501-504

[2].Gonzalez R. C., Woods R. E., Digital Image Processing, Second Edition, ISBN: 0-20-118075-8

[3]. Xiang, T., Qu, J., \& Xiao, (2014). Joint SPIHT compression and selective encryption. Applied Soft Computing, 21, 159-170.

[4]. S.P.Raja, Dr.A. suruliandi,"Analysis Of efficient wavelet based Image Compression Techniques, "Second International Conference on Compression Using Communication networking Technology,

[5]. D.Taubman and M.W.Marcelin,"JPEG 2000 Image Compression Fundamentals Standards and practice", Dordrecht the Netherlands: Kluwer.

\section{AUTHORS PROFILE}

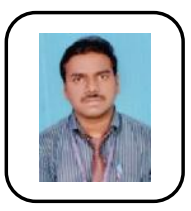

\section{Mr.Nithin S.S,}

Educational Profile:

Doing Ph.D- Noorul Islam University

ME-Communication System

BE-Electronics and Communication Engineering,

Teaching Experience: 8 Years

Area of Specialization:Image Processing, Published various papers

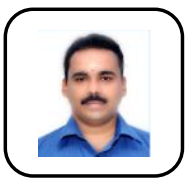

Dr. L Padma Suresh.

received his B.E. Electrical and Electronics Engineering from Noorul Islam College of Engineering on 1999 and M.E. Process Control and Instrumentation in 2000 from Annamalai University and Ph.D. Electrical Engineering in 2010 from Dr.M.G.R University and Ph.D Computer Science Engineering from M.S. University. He has 18years of diverse experience in teaching , Life member of ISTE, and Senior IEEE Member. His Research interests in Artificial Intelligence, Control Systems, Power Electronics and Image Processing. 